\title{
Optimizing engineered TCR T cell therapy for synovial sarcoma
}

\author{
Sandra D'Angelo' ${ }^{1}$, Melinda Merchant ${ }^{2}$, Luca Melchiori ${ }^{3}$, Hua Zhang ${ }^{2}$, Lini Pandite ${ }^{4}$, Tom Holdich³, \\ Gwendolyn Binder-Scholl ${ }^{4}$, William Tap ${ }^{1}$, Marylene Fortin ${ }^{5}$, Yoav Peretz ${ }^{5}$, Matthew Wright ${ }^{2}$, Paul Meyers ${ }^{1}$, \\ Rafael Amado ${ }^{4}$, Bent Jakobsen ${ }^{3}$, Crystal Mackall ${ }^{*}$
}

From 30th Annual Meeting and Associated Programs of the Society for Immunotherapy of Cancer (SITC 2015) National Harbor, MD, USA. 4-8 November 2015

Relapsed or metastatic synovial sarcoma remains a significant unmet medical need. NY-ESO-1 is an attractive target for sarcoma, since it is expressed in approximately $70 \%$ of synovial sarcomas but not on vital tissues. We generated NY-ESO ${ }^{\text {c259 }}$, a human-derived affinity-enhanced T-cell receptor (TCR) that recognizes the NY-ESO-1- derived SLLMWITQC peptide in complex with HLA-A*02. Adoptive transfer of autologous T cells expressing NY-ESO ${ }^{\text {2259 }}$ was clinically tested in patients with metastatic synovial cell sarcoma and melanoma whose tumor expressed NYESO-1 protein at an intensity of $\geq 2+$ and in $50 \%$ of cells by immunohistochemistry; infusion followed preconditioning with fludarabine and cyclophosphamide, and systemic IL-2 support was given [1]. We subsequently initiated a study in relapsed/metastatic synovial sarcoma, with an updated $\mathrm{T}$ cell manufacturing method utilizing anti-CD3/28 paramagnetic microbeads for simultaneous activation and co-stimulation of $\mathrm{T}$ cells, and genetic modification with a lentivector (NCT01343043). No IL-2 support was given. Outcomes of this study were recently presented[2]; clinical data demonstrate safety, objective clinical responses in $50 \%$ of patients, and improved and durable engineered $\mathrm{T}$ cell persistence. This study has been extended to include two additional cohorts to evaluate the removal of fludarabine on tumor responses (Cohort 3) and to evaluate responses in patients who have antigen positive tumor below the current $\geq 2+$ and $50 \%$ threshold (Cohort 2).

To better understand the lineage and functional characteristics of the persisting engineered $\mathrm{T}$ cells, as well as to understand the role of the starting material and manufacturing method in modifying the $\mathrm{T}$ cell phenotype and its fate once in vivo, we performed multiparameter flow analysis. Baseline and post infusion PBMCs, as well as manufactured product, were analyzed to evaluate memory and exhaustion markers (e.g. CD45RA, CCR7, PD-1), polyfunctionality/cytotoxicity markers (e.g. IFN- $\gamma$ and Granzyme B), and costimulatory markers (e.g. OX40, ICOS, CD28). TCR expression was measured by pentamer. Hierarchical cluster analysis was applied to identify trends of expression of surface markers and their correlation with final cell product profile, persistence and clinical response. An update of Cohort 1 safety, efficacy and correlative biomarker analyses, and a status update on Cohorts 2 and 3 will be presented.

\section{Trial registration}

ClinicalTrials.gov identifier NCT01343043.

\begin{abstract}
Authors' details
${ }^{1}$ Memorial Sloan Kettering Cancer Center, New York, NY, USA. ${ }^{2}$ National Cancer Institute, Bethesda, MD, USA. ${ }^{3}$ Adaptimmune, Abingdon, United Kingdom. ${ }^{4}$ Adaptimmune, Philadelphia, PA, USA. ${ }^{5}$ Immunecarta Services, Montreal, USA.
\end{abstract}

\section{Published: 4 November 2015}

\section{References}

1. Robbins PF, et al: A pilot trial using lymphocytes genetically engineered with an NY-ESO-1-reactive T cell receptor: Long term follow up and correlates with response. Clinical Cancer Research 2015, 21(5):1019-27.

2. Merchant SMelinda, et al: Genetically engineered NY-ESO-1 specific T cells in HLA-A201+ patients with advanced cancers. American Society of Clinical Oncology Chicago, Illinois; 2015.

\section{doi:10.1186/2051-1426-3-S2-P159}

Cite this article as: D'Angelo et al:: Optimizing engineered TCR T cell therapy for synovial sarcoma. Journal for ImmunoTherapy of Cancer 20153 (Suppl 2):P159.

${ }^{2}$ National Cancer Institute, Bethesda, MD, USA

Full list of author information is available at the end of the article 